\title{
GeMS: first on-sky results
}

\section{François Rigaut, Benoit Neichel, Maxime Boccas, Céline d'Orgeville, Gustavo Arriagada, et al.}

François Rigaut, Benoit Neichel, Maxime Boccas, Céline d'Orgeville, Gustavo Arriagada, Vincent Fesquet, Sarah J. Diggs, Claudio Marchant, Gaston Gausach, William N. Rambold, Javier Luhrs, Shane Walker, Eleazar Rodrigo Carrasco-Damele, Michelle L. Edwards, Peter Pessev, Ramon L. Galvez, Tomislav B. Vucina, Claudio Araya, Alejandro Gutierrez, Angelic W. Ebbers, Andrew Serio, Cristian Moreno, Cristian Urrutia, Rolando Rogers, Roberto Rojas, Chadwick Trujillo, Bryan Miller, Douglas A. Simons, Ariel Lopez, Vanessa Montes, Herman Diaz, Felipe Daruich, Felipe Colazo, Matthieu Bec, Gelys Trancho, Michael Sheehan, Peter McGregor, Peter J. Young, Matthew C. Doolan, Jan van Harmelen, Brent L. Ellerbroek, Damien Gratadour, Aurea Garcia-Rissmann, "GeMS: first on-sky results," Proc. SPIE 8447, Adaptive Optics Systems III, 84470I (13 September 2012); doi: 10.1117/12.927061

Event: SPIE Astronomical Telescopes + Instrumentation, 2012, Amsterdam, Netherlands 


\title{
GeMS first on-sky results
}

François Rigaut ${ }^{a, b}$, Benoit Neichel ${ }^{a}$, Maxime Boccas ${ }^{a}$, Céline d'Orgeville ${ }^{a, b}$, Gustavo Arriagada $^{a}$, Vincent Fesquet ${ }^{a}$, Sarah J.Diggs ${ }^{a}$, Claudio Marchand ${ }^{a}$, Gaston Gausachs ${ }^{a}$, William N.Rambold ${ }^{a}$, Javier Lurhs ${ }^{a}$, Shane Walker ${ }^{a}$, Eleazar Rodrigo Carrasco-Damele ${ }^{a}$, Michelle L.Edwards ${ }^{a}$, Peter Pessev ${ }^{a}$, Ramon L.Galvez ${ }^{a}$, Tomislav B.Vucina ${ }^{a}$, Claudio $\$ \mathbb{D} \mathrm{D}^{a}$, Alejandro Gutierrez ${ }^{a}$, Angelic W.Ebbers ${ }^{a}$, Andrew Serio ${ }^{a}$, Cristian Moreno ${ }^{a}$, Cristian Urrutia ${ }^{a}$ , Rolando Rogers ${ }^{a}$, Roberto Rojas ${ }^{a}$, Chadwick Trujillo ${ }^{a}$, Brian Miller ${ }^{a}$, Douglas A.Simons ${ }^{a}$, Ariel Lopez ${ }^{a}$, Vanesa Montes ${ }^{a}, \operatorname{Herman~Diaz}^{a}$, Felipe Daruich $^{a}$, Felipe Colazo ${ }^{a}$, Matthieu Bec $^{c}$, Gelys Trancho ${ }^{c}$, Michael Sheehan ${ }^{c}$, Peter McGregor ${ }^{b}$, Peter J.Young ${ }^{b}$, Matthew C.Doolan ${ }^{b}$, Jan van Harmelen ${ }^{b}$, Brent L.Ellerbroek ${ }^{d}$, Damien Gratadour ${ }^{e}$, Aurea Garcia-Rissmann ${ }^{f}$

${ }^{a}$ Gemini Observatory, c/o AURA, Casilla 603, La Serena, Chile;

${ }^{b}$ Australian National University, Research School of Astronomy and Astrophysics, Mount

Stromlo Observatory, Cotter Road, Weston ACT 2611, Australia;

${ }^{c}$ Giant Magellan Telescope Org. Corp., PO box 90933, Pasadena, CA, 91109, USA;

$d$ Thirty Meter Telescope, 1200 E. California Blvd., Pasadena, CA 91125, USA;

${ }^{e}$ LESIA, Observatoire de Paris-Meudon, place Jules Janssen, 92190 MEUDON, France;

${ }^{f}$ European Southern Observatory, Karl-Schwarzschild-Str. 2, 85748 Garching bei München, Germany

\begin{abstract}
GeMS, the Gemini Laser Guide Star Multi-Conjugate Adaptive Optics facility system, has seen first light in December 2011, and has already produced images with H band Strehl ratio in excess of $35 \%$ over fields of view of $85 \times 85$ arcsec, fulfilling the MCAO promise. In this paper, we report on these early results, analyze trends in performance, and concentrate on key or novel aspects of the system, like centroid gain estimation, on-sky noncommon path aberration estimation. We also present the first astrometric analysis, showing very encouraging results.
\end{abstract}

Keywords: Multi-Conjugated Adaptive Optics, Adaptive Optics performance, astrometry

\section{INTRODUCTION}

GeMS is a Multi-Conjugate Adaptive Optics (MCAO) system in use at the Gemini South telescope. It uses five Laser Guide Stars (LGS) feeding five 16x16 Shack-Hartmann for an image compensation in the near infrared. Its dedicated imager, GSAOI, covers a field of view of $85 \times 85$ arcsec using four $2 \mathrm{kx} 2 \mathrm{k}$ Hawaii2RG detectors. Tip-tilt (TT) and plate scale modes compensation can be fed by either three visible-sensitive TT sensors (located inside CANOPUS, the GeMS optical bench) or three out of four possible On-Detector Guide Windows (ODGW) available directly in the GSAOI science focal plane. GeMS, CANOPUS, its laser system, associated subsystems and GSAOI have been extensively described in previous publications. ${ }^{1-6}$ Description of the concepts and limitations of MCAO can be found in the following references. ${ }^{7,8}$

Commissioning of GeMS started in January 2011, and is now (June 2012) reaching completion. It should be available for science verification at the end of 2012 and open to the community in 2013. The commissioning schedule, as well as many other aspects of operation and instrument performance, are developed in more details in a companion paper. ${ }^{9}$ Still more papers report on other aspects, as laser, Beam Transfer Optics and launch systems,${ }^{10}$ Sodium layer content,${ }^{11}$ Interaction matrices calibrations, ${ }^{12}$ and GSAOI commissioning. ${ }^{13}$

Send correspondence to frigaut@mso.anu.edu.au or bneichel@gemini.edu

Adaptive Optics Systems III, edited by Brent L. Ellerbroek, Enrico Marchetti, Jean-Pierre Véran, Proc. of SPIE Vol. 8447 , 84470I - (C) 2012 SPIE · CCC code: 0277-786/12/\$18 · doi: 10.1117/12.927061 


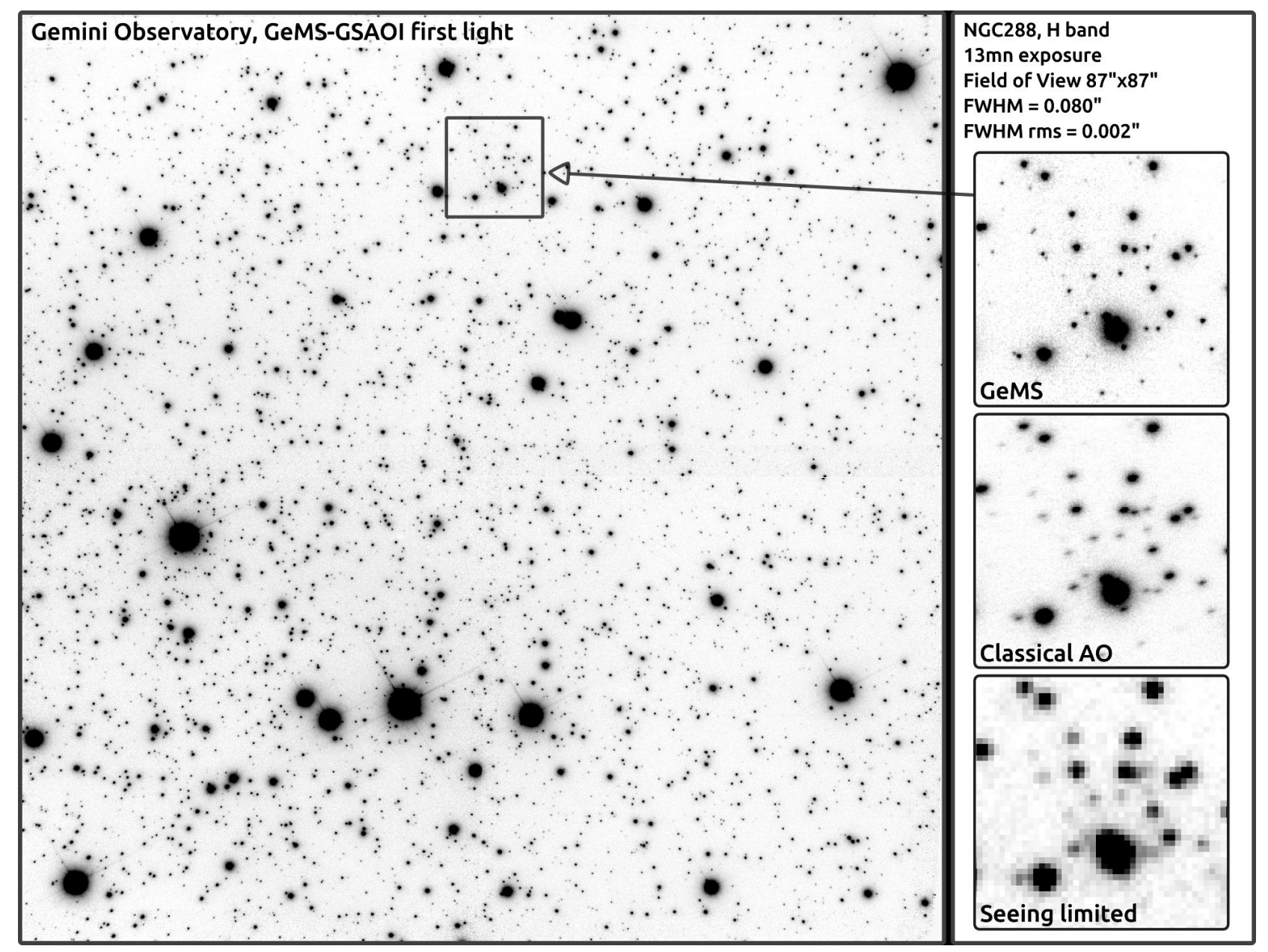

Figure 1. NGC288 at H band, taken with GeMS + GSAOI on December 17, 2011. The field of view is $87 \mathrm{x} 87$ ". This image is a combination of thirteen 15 seconds images. The FWHM varies between 70 and 85 mas over the field of view. The Strehl is approximately $20 \%$ (see section 2.1 for variability). This image is typical of performance obtained under median or slightly better than median seeing conditions. The insets on the right show an enlarged version of the marked sub-region, for GeMS (up), a seeing limited case (bottom), and a classical AO version, assuming the natural guide star is the bright star on the upper right corner of the field. Note that the pixel size on the seeing limited image has been choosen to maximize signal to noise ratio per pixel, while not degrading angular resolution (Nyquist sampling the seeing images).

The goal of this paper is to present the very first performance results of GeMS, which is the first -and only to date- MCAO system using LGS. MAD at ESO pioneered MCAO for nighttime astronomy, ${ }^{14}$ but was using NGS. Compared to NGS systems, systems using LGS provide more consistent image quality uniformity and larger sky coverage. The results presented here are based on data obtained throughout several runs at the end of 2011 and the start of 2012, with a special focus on the run of December 2011, during which the very first images were recorded, and the weather and seeing were cooperating.

In this paper, we report on image quality, in term of FWHM and Strehl ratio, and field uniformity thereof. We give the first results of an analysis of astrometric performance. Finally, we describe new issues that have been uncovered during the commissioning and the solutions that were adopted to solve them. 


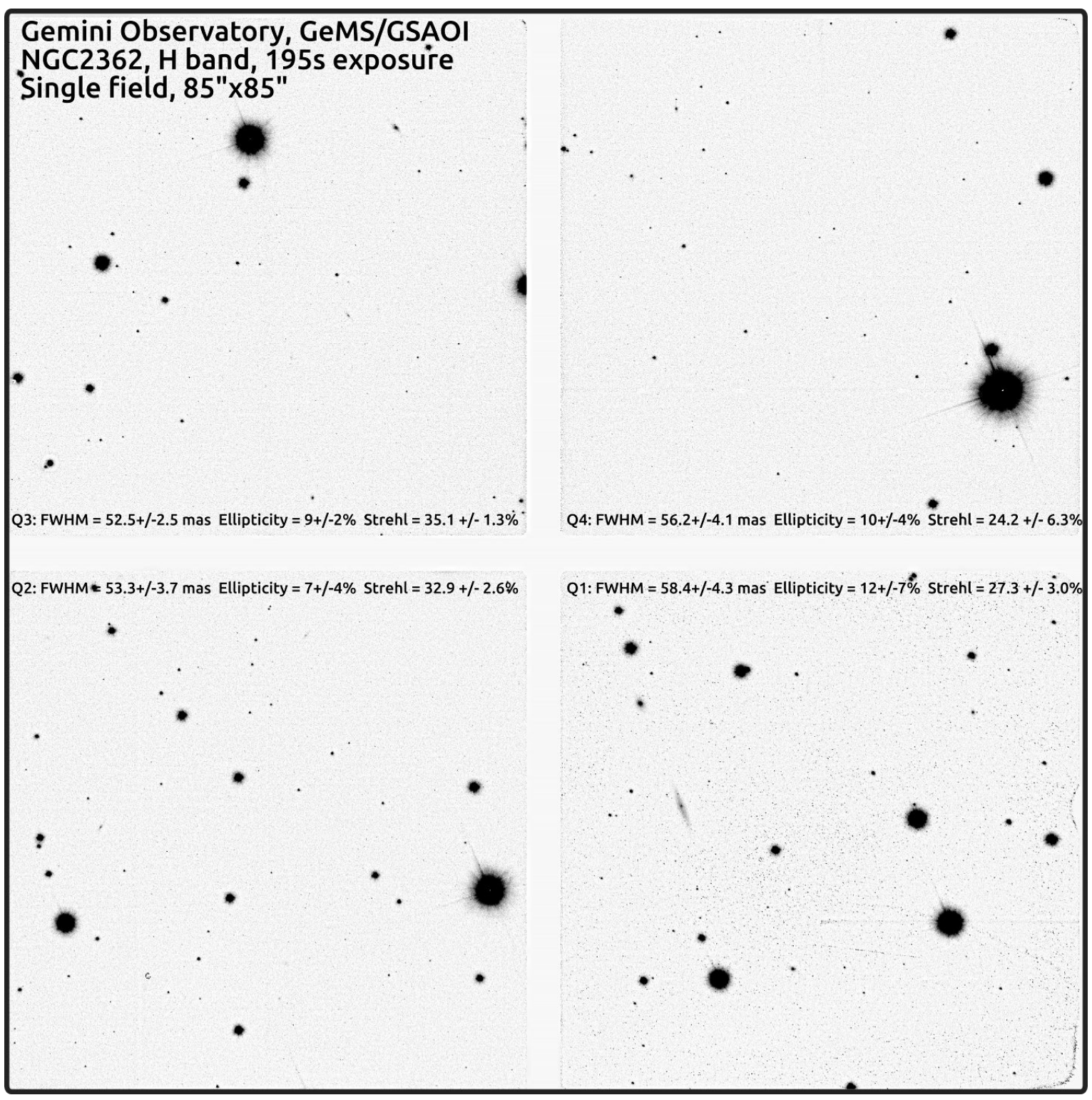

Figure 2. NGC2362 at H band, taken with GeMS + GSAOI. The field of view is 85x85" (the white cross is the gaps between the HAWAII 2RG arrays). This image is a combination of fifteen 15 seconds exposures, acquired over the course of 2 hours (no exposure selection was performed), during technical tests on December 18, 2011. The FWHM and Strehl ratio (average and standard deviation over each array) are reported on the figure. In some images, Strehl as high as $40 \%$ were measured, which we believe make this image the highest $\mathrm{H}$ band Strehl ratio image obtained on a large telescope with a $L G S$ AO system (MCAO solves implicitly for the cone effect).

\subsection{Ultra-short commissioning summary and a note on data validity}

The GeMS commissioning started in January 2011. Functional commissioning went through May 2011, followed by a planned shutdown in the austral winter, less favorable to LGS-based observations. ${ }^{9}$ Sky commissioning proceeded as scheduled in November 2011, focusing primarily on performance characterization and optimization, as well as the commissioning of GSAOI. As shown below, the first science data were obtained in December 2011. Various weather related and technical issues were encountered during the next runs (GSAOI cooling issue, LGS constellation alignment). Starting in January 2012, a LGS WFS misalignment issue also reduced performance. 
Unfortunately, hidden by other issues, it was not recognized as such until the April run. The bottom line is that the December data are right now the most representative of the final system, as at this date the almost entirety of the subsystems were commissioned and functioning as expected (with some exceptions). The data presented in this paper are from the December 2011 and the April 2012 runs (GSAOI). Some GMOS data from the March 2012 run are also presented. As far as seeing, it was median (e.g. NGC288) to exceptional (e.g. NGC2362) during the December period. The Sodium content, though, was close to its minimum, due to seasonal variations. ${ }^{12}$

In the data presented in this paper, we have avoided using data selection as most as possible. All average images were produced without images selection, unless specified. Our experience is that the NGC288 data, for instance, are fairly typical of what GeMS can deliver under median seeing and decent sodium return conditions (140 to 200 detected photons/subaperture/frame at $200 \mathrm{~Hz}$ ).

We realize however that, being based on a handful of data sets, the results of this paper are somewhat anecdotal. We are planning to perform a more systematic performance analysis, based on many more data, when these will become available.

\section{IMAGING PERFORMANCE}

Although there were spectroscopy applications in the GeMS science case, the main science driver for GeMS is in wide field imaging. GSAOI ${ }^{13}$ is the main instrument for GeMS. It is a $4 \mathrm{kx} 4 \mathrm{k}$ imager covering $85 \mathrm{x} 85 \mathrm{arcsec}$ at 20 milliarcsec/pixel. It has a full featured filter set, covering the $\mathrm{z}$ through $\mathrm{K}$ band wavelength ( 0.9 to 2.5 microns). It uses on-axis refractive optics and has essentially no optical aberrations. Here, we report on performance in term of compensated image quality: FWHM, Strehl ratio and uniformity thereof.

Figure 1 is the GeMS scientific first light image. It was acquired on December 17, 2011, and is made of thirteen 15 seconds $\mathrm{H}$ band images (1.65 microns wavelength). It shows a part of the globular cluster NGC288. The high order and the TT loops were closed, while the plate scale loop was not. The FWHM is about 80 mas over the vast majority of the field of view and the Strehl hovers at about $20 \%$. Seeing was median or slightly better during the acquisition.

Figure 2 was acquired the next night, on December 18, 2011. It shows the open cluster NGC2362 in the $\mathrm{H}$ band. There are fifteen images of 15 seconds in the data set, acquired over the course of over 2 hours. All loops, including the plate scale loop, were closed. The seeing was very good during the acquisition ( 0.35 arcsec or better). The FWHM and Strehl are surprisingly uniform over the field of view (see below). The best images of the data set have FWHM values lower than 50mas and Strehl ratio in excess of $40 \%$, which we believe makes these the best images obtained to date with a $L G S \mathrm{AO}$ system at $\mathrm{H}$ band (without even mentioning the fact that the correction extend over 85 arcsec). Generally, for AO systems on 8-m class telescopes, $\mathrm{H}$ band performance suffers from significant focus anisoplatism error (cone effect). At the expense of generalized fitting (see section 2.2 and $\mathrm{ref}^{8}$ ), the latter is naturally solved by MCAO, through its tomographic reconstruction process.

Last, Figure 3 shows a single 600 second exposure of the planetary nebula NGC6369 acquired with GMOS in the red, at I band (between 800 and $850 \mathrm{~nm}$ wavelength, as the I band is interupted by the CANOPUS dichroic in the short wavelength part of the spectrum). Using GMOS with CANOPUS has never been very high in the observatory priorities as it was believed performance was going to be marginal. This image, with about 80 milliarcsec FWHM over most of the 2 arcmin field of view unvignetted by CANOPUS, proves that when the seeing cooperates, GeMS can deliver down to the frontier of the red part of the visible spectrum. This image was our best with GMOS though. Under median seeing conditions, GeMS provided typically a factor of 2 to 2.5 improvement in FWHM, which is roughly what was expected (i.e. slightly better than GLAO).

\subsection{Uniformity}

Figure 4 shows iso-contours of FWHM (top) and Strehl ratio (bottom) for NGC288 and NGC2362, and a data set acquired on the Galactic center region on April 2012 (four images). As stated above, NGC288 and NGC2362 are at $\mathrm{H}$ band (1.65 microns). The Galactic center data set was acquired with the Ks filter (2.15 microns).

For each data set, PSF fitting was performed for as many stars in the field as available: Close to 1000 for NGC288 and the Galactic Center and about 100 for NGC2362. For each image of the data set, FWHM and Strehl 


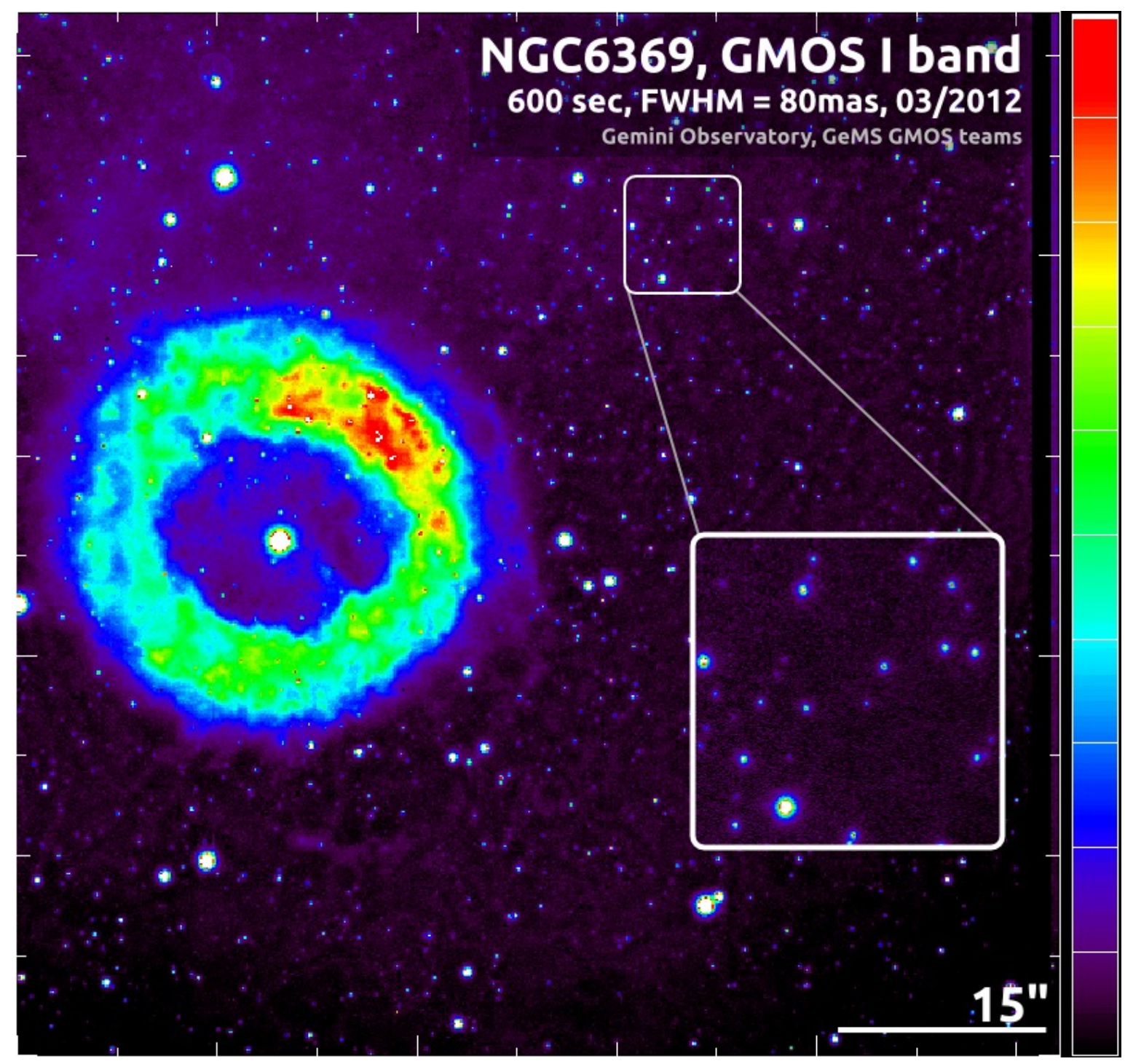

Figure 3. NGC6369 at $\approx 830 \mathrm{~nm}$, taken with GeMS + GMOS on March 14, 2012. The field of view is $75 \times 75$ arcsec, an excerpt of the 2 arcmin diameter field of view passed by GeMS. Exposure time is 10mn. The natural seeing was between 0.3 and 0.4 ", the FWHM of the corrected images over the displayed field is between 80 and 90 milliarcsec. Pixels have been rebinned on the large image to improve the visibility of faint extended structures. The image insert shows the raw (unrebinned) image with the 36 mas pixels. Note that the detector exhibits quite a bit of fringing at this wavelength which was not corrected in this image.

maps can thus be drawn. The maps shown in Fig 4 are averaged over all data set images. Because of crowding, a fairly simple PSF model using a Lorentzian-type one parameter function was used $\left(f(r)=g^{2} /\left(g^{2}+r^{2.4}\right)\right)$ which was deemed to fit the data acceptably. This functional form links the Strehl ratio and the FWHM estimation, explaining the similarity of the FWHM and Strehl ratio maps for a given data set. This method lacks the accuracy of a full fledge AO-type PSF fitting methods, but is much more robust in crowded fields like the ones used here.

As shown by the plots, the FWHM is relatively uniform in the major fraction of the fields of view. Within the best $60 \times 60$ arcsec section of the $85 \times 85$ arcsec field of view, the FWHM has variations with peak to peak of 8 mas for NGC2362 ( $\mathrm{rms} \approx 2 \mathrm{mas}$ ), $11 \mathrm{mas}$ for NGC288 (rms $\approx 2.5 \mathrm{mas}$ ) and 20mas for the Galactic Center (rms $\approx 3.5 m a s)$, which are compatible with the expected performance in term of variability. That's about $4 \%$ relative 
rms variation, a vast improvement over what can be achieved with single-conjugate AO systems.

Factors that degrade the image quality differentially in the field of view are high order turbulence residuals, anisokinetism residuals and field-varying static aberrations. Depending on the data set, one or the other might dominate.

Note that these maps are generally not exactly centered on the TTGS constellation. This probably indicates that the Tip-Tilt residuals are not the dominant factor, unless the wind direction and servolag shifts the TT map minimum away from the TT guide star constellation's center (NGC288 was taken with a high order and TT loops frequency of $200 \mathrm{~Hz}, \mathrm{NGC} 2362$ of $250 \mathrm{~Hz}$ and the GC of $300 \mathrm{~Hz}$ ). The most likely source of variation, though, is linked to static aberration variations, resulting from the LGS WFS internal aberrations combined with the centroid gains estimation errors, as explained in section 2.4. Not reported here is the fact that these maps are generally not stable from images to images. This could also naturally be explained by the same centroid gain issue.

Interestingly, in the NGC2362 data set, the LGS 4 (lower right) was not used, following a technical problem with one of the LGS WFS mechanism. Even though the performance is still decent in this lower right image section, the maps clearly show some performance degradation.

\subsection{The MCAO PSF}

It was mentioned above that a Lorienzian type of profile was found to fit the PSF the best. Figure 5 shows an example profile for the NGC288 image. The MCAO PSF, and the MCAO error budget for that matter, is different than for regular single conjugate AO..$^{8,15}$
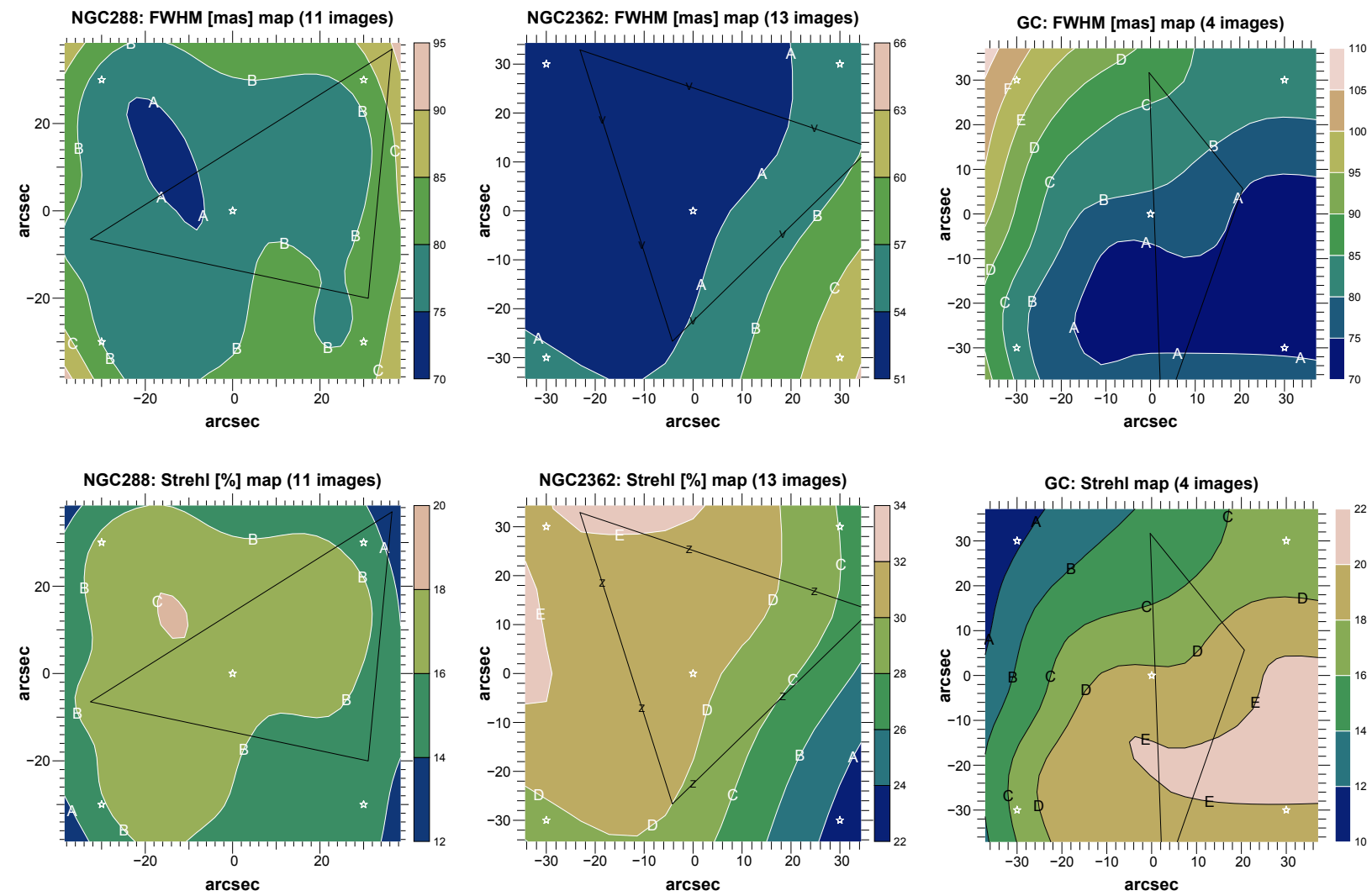

Figure 4. FWHM (top) and Strehl ratio (bottom) maps for three data sets obtained in December 1011 and April 2012 , showing the variation of these quantity in the GSAOI field of view. NGC288 and NGC2362 are at H band, the GC is in the Ks band. The triangle marks the Tip-Tilt natural guide star constellation. 
The main difference comes from a term dubbed, according to the authors, generalized anisoplanatism, generalized fitting or tomographic error (although the last one has a slightly different scope as it includes generally all tomographic related error, including reconstruction errors). Here, we will use generalized fitting.

Generalized fitting is a result of the fact that the phase corrector is not continuous (in altitude), but instead made of a finite number of deformable mirrors, conjugated to discrete altitudes: In GeMS, the design was for $3 \mathrm{DMs}$ but following issues with DM0 we are temporarily using $2 \mathrm{DMs}$ at 0 and $9 \mathrm{~km}$ altitude. Because the correction is effected over a finite field of view, the system is not capable of correcting the perturbations at the cut-off frequency of the DMs over the whole column of turbulence. This was illustrated very neatly by Ragazzoni and the layer-oriented group. ${ }^{15}$ As the distance of a layer to the nearest DM increases, the fitting error increases, hence the term generalized fitting. The residual phase error is thus a superposition of residual phases that have a different fitting error depending on the altitude at which they originated. It can readily be understood that the final performance will depend on the actual vertical distribution of turbulence. The net result is not unlike the effect of partial correction in AO, with a slightly different twist. The PSF is almost always of a Lorentzian shape (see figure5), with a narrow central core but without well defined airy rings nor the well defined dual core-halo shape usual to AO correction. This is indeed generally what we find in GeMS images, except when the seeing was exceptional (e.g. NGC2362), although even in that case, for which the Strehl ratio was close to 40\%, the first airy ring is visible but not conspicuous.
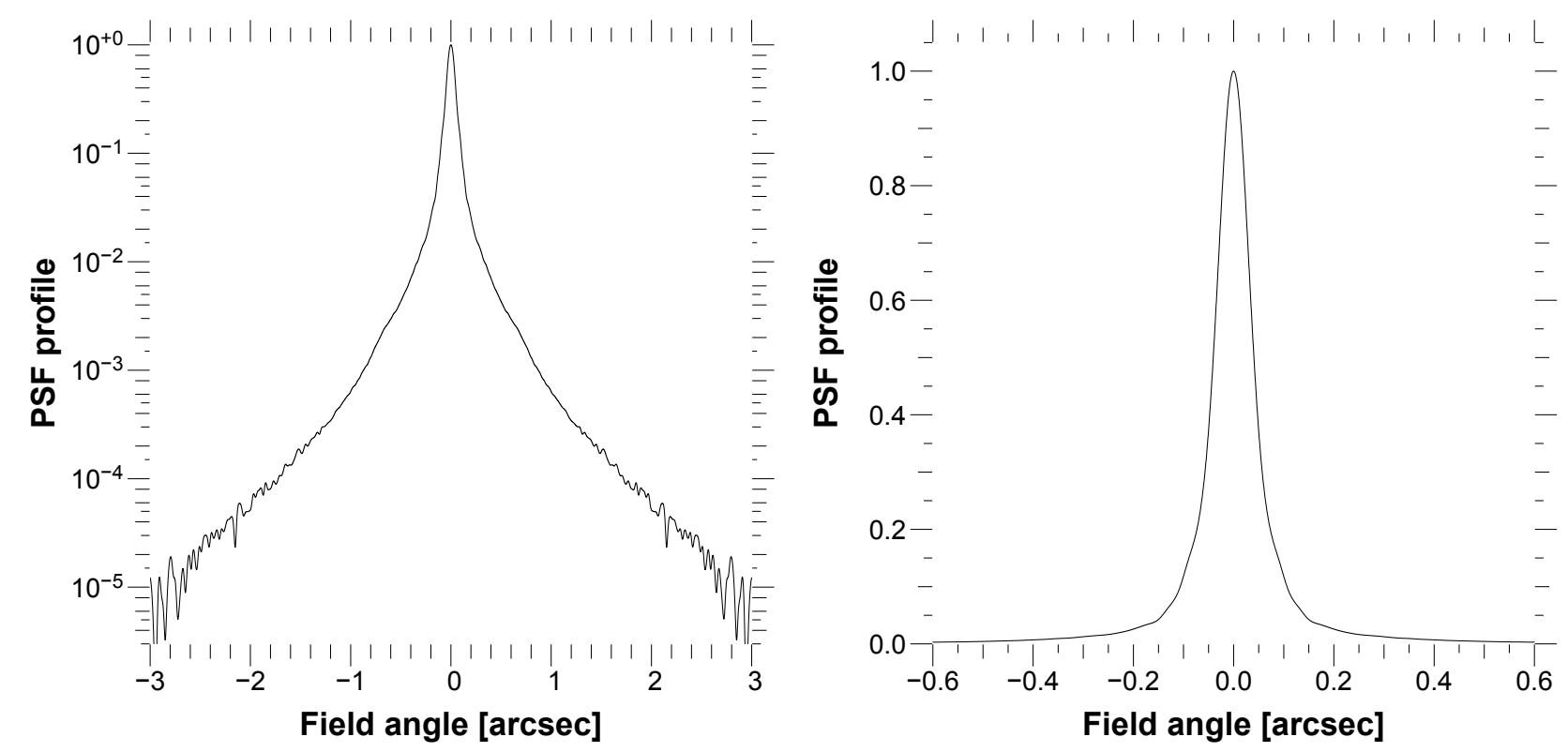

Figure 5. PSF profile extracted from the NGC288 data. Left: Lin-Log, Right: Lin-Lin.

\subsection{Preliminary phase-based error budget}

In GeMS, we have the ability to record the system telemetry by burst, the so-called "circular buffers", that contains all system data for period of 30 seconds and can be saved for off-line analysis.

Early examination of these buffers showed an excess of power at low frequency in the modal coefficients (e.g. Zernike) temporal Power Spectral Density (PSD). This feature was attributed to the generalized fitting error, and we developed a theoretical approach to determine the split between noise, servolag and generalized fitting in the modal coefficients temporal PSDs. Briefly, this approach is based on the fact that the tomographic/generalized fitting error is filtered by the reconstructor and thus is not affected by the close loop transfer functions. Hence, its high frequency part (essentially, the noise) will be flat, while the high frequency part of the servolag will be shaped by the error transfer function. Using this method lead to sample results presented in the table below. The data were obtained in March 2012. Case 1, 2 and 3 cover a range of seeing; respectively bad, median and good. The loop sampling frequency, the $0 \mathrm{bD}$ point of the error transfer function are reported, together with 
the error budget, splitting the servolag, generalized fitting and noise contributions, in nanometers. The numbers between parenthesis are the associated Strehl ratio values at $\mathrm{H}$ band, using the simple Marechal approximation. Note that this covers only part of the total error budget: not included is fitting, TT and plate scale modes and static aberrations, to cite only the most important additional contributors. However, the Strehl computed from the total of the three error considered here (last line) are roughly in line with what has been obtained on sky from the GSAOI images.

$\begin{array}{llll}\text { Case } & 1 & 2 & 3 \\ \text { Sampling [Hz] } & 300 & 300 & 400 \\ \text { OdB BW, ETF [Hz] } & 17.3 & 26.7 & 41.4 \\ \text { Uncorrected [nm] } & 1227(0 \%) & 950(0 \%) & 756(0 \%) \\ \text { Servolag [nm] } & 258(38 \%) & 140(75 \%) & 92(88 \%) \\ \text { Gen. Fitting [nm] } & 332(20 \%) & 314(24 \%) & 184(61 \%) \\ \text { Noise [nm] } & 84(90 \%) & 68(93 \%) & 36(98 \%) \\ \text { Total [nm] } & 429(7 \%) & 350(17 \%) & 209(53 \%)\end{array}$

These results, if confirmed with further data and analysis, indicate that GeMS is currently limited by generalized fitting, i.e. we are suffering more than expected from the lack of mid-altitude layer. Note that these data were obtained with the default MMSE reconstructors, but tests using LSE reconstructors did not result in drastically different conclusions (the generalized fitting was slightly lower, but stability was affected -read, not as good as when using a MMSE- in most of the cases).

This is encouraging in the sense that we can expect significant improvements when the mid-altitude DM, currently being repaired, will be fitted back into the system, almost doubling the number of active actuators available.

\subsection{Performance Limitations}

There are several factors currently limiting compensation performance. In order of severity, these are:

- The main factor is photon return. Currently, we project about $4 \mathrm{~W}$ of $589 \mathrm{~nm}$ in each beam/LGS. ${ }^{9,10}$ The laser spectral format has a relatively low coupling efficiency with the sodium atoms, ${ }^{12}$ and thus in low to medium sodium season, the system works typically with about 140 photons/subaperture/frame at $200 \mathrm{~Hz}$. This corresponds to 35 photons/pixel/frame (the LGS WFS uses quadcells and has a read out noise of 3 to 4 electrons), which we have determined to be the minimum we can work with before performance and stability start to be critically affected. $200 \mathrm{~Hz}$ of operating frequency is very low (the system was designed and can work up to $800 \mathrm{~Hz}$ ), leading to a large servolag error.

- From the analysis presented above, it seems that generalized fitting (tomographic error) is significant. Following technical problems with one of the deformable mirror, CANOPUS currently uses only two DMs (at 0 and $9 \mathrm{~km}$ with 0.5 and $1-\mathrm{m}$ pitch resepectively) instead of the three initially planned $(0,4.5$ and $9 \mathrm{~km}$ with $0.5,0.5$ and $1-\mathrm{m}$ pitch respectively). This reduced the number of active actuators from 684 (design) down to 360 (current). Even though the performance will not simply scale with the number of actuators (the missing ones were aimed to deal with mid-turbulence layers where there is less turbulence than on the ground for instance), it can be easily conceived that this will reduce performance in most of the cases, and will certainly make the system performance less robust to changes in the $\mathrm{Cn} 2$ profile. In effect, the current two DMs system can be viewed as a fairly potent GLAO system (17x17 actuators across M1) with an additional low order DM at altitude (9x9 actuator across M1).

- Static aberrations are still currently an important issue. These are mostly a result of the combination of relatively large LGS WFS internal aberrations (differential from beam to beam, for instance there is $600 \mathrm{~nm}$ of differential focus between the central and the upper right corner LGS WFS, while astigmatism typical variations from WFS to WFS is close to $100 \mathrm{~nm}$ ). To avoid introducing them into the compensated images, these aberrations have to be subtracted before reconstruction, thus requiring a good estimate of the LGS WFS centroid gains. Typically, 5 to $10 \%$ would be required. We are not there yet (see section 4.1 ). 
The limitation in limiting magnitude of the natural guide stars are discussed in another paper: ${ }^{9}$ Fiber injection problems limit the natural guide star magnitude to about 16.5, significantly reducing the sky coverage. Operational limitations also have a significant impact and have been described in the same companion paper. ${ }^{9}$

\section{ASTROMETRIC PERFORMANCE}

How well MCAO fares as far as astrometry has been the subject of many discussions. Potentially, MCAO can be a very powerful astrometric tool, providing access to large fields of view with the increased astrometric accuracy directly resulting from the improved FWHM brought by AO compensation (see for instance the 300 microarcseconds achieved by the UCLA group on the galactic center images). There are however potential pitfalls: MCAO having the ability to compensate for plate scale and dynamic atmospheric-induced field distortions, it also has the capability to mess it up, if anything goes wrong. For instance, an actuator sticking out -for whatever reason- in a DM conjugated to an non-zero altitude will induce local plate scale distortions that will affect and potentially destroy the astrometry. It was for this reason, and the lack of experimental data, that no astrometry science cases were included in the GeMS science case.

In the December 2011 commissioning run, the team acquired eleven 15 seconds images on NGC2362 during the course of image quality stability tests. These images were acquired on the course of over approximately 35 minutes. As the intent was not to produce the best science images, no spatial dithering was used, thus minimizing the impact of static distortion errors. This makes this data set ideal for investigating the GeMS astrometric properties. FWHM was typically very uniform at 55mas and Strehl ratio was between 25 and $40 \%$ PTV across the data set.
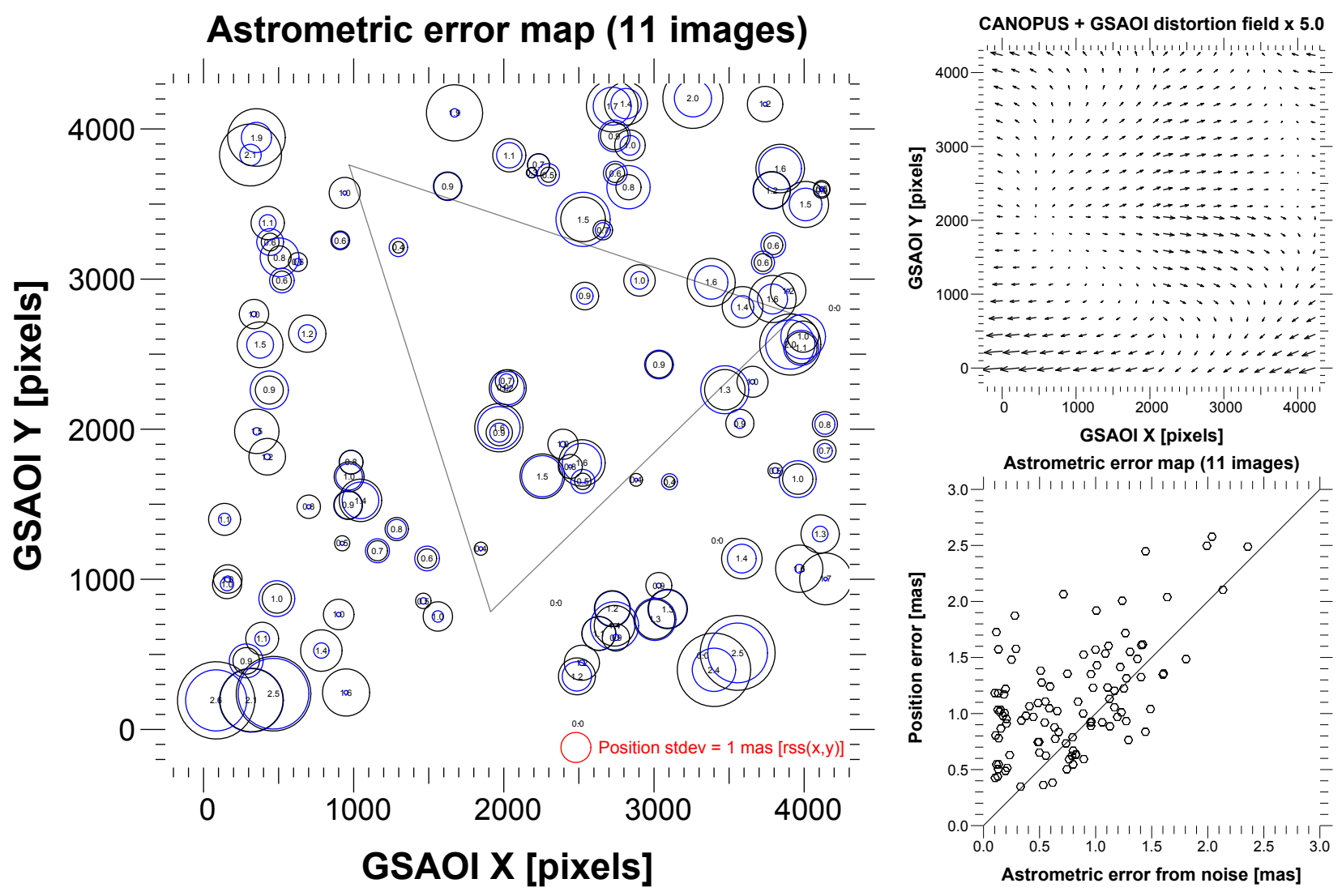

Figure 6. Left: Astrometric error: circles plot the astrometric error versus position in the field. The blue circles are the expected error due to photon noise. The triangle shows the location of the NGS constellation. The field of view is $85 \times 85$ arcsec. Top Right: Static distortion field $\times 5$. Bottom Right: Position error vs position error as expected from photon noise. 
The image data reduction performed on the data set images is standard; images were sky subtracted, flat fielded, cosmic rays and dead pixels corrected (using nearest neighbor interpolation). Note that because dither was not used, we had to use a sky from the previous night, which is obviously sub-optimal for our astrometric analysis.

This field in NGC2362 is quite sparse, which is well suited for our astrometric application. 112 unsaturated stars above a certain threshold (about $200 \mathrm{ADU} / 15$ seconds) were selected. The astrometric data reduction has been carried out as follow. First for each image, the 112 stars positions were determined by correlating with the field-averaged PSF. Because of distortion "drifts" and other limitations exposed in section 3.1, star positions for each images were "recentered" with respect to a common reference using 6 modes: X and Y offsets (2), rotation (1) and the plate scale modes (3). Doing this does not significantly affect the final position rms we are interested in (6 parameters model to fit $112 \times 2$ variables). Note that this was performed using all stars, irrespective of -and unweighted by- their brightness.

We then used these 11x112 star positions for two analysis: To compute the rms positions of each stars, and to compute the rms separations of all star pairs. The latter is the most relevant, but the former will give indications about any field dependence of the astrometric error.

Fig 6 presents the results of the position rms for each star along the image data cube (black circles). The blue circles are the position errors expected from noise, using FWHM $/ \sqrt{\mathrm{N}_{\mathrm{ph}}}$ as an estimator of the position error from photon noise only. A few comments:

- Although the distribution of errors is quite complex, there is a tendency for it to be larger outside of the Tip-Tilt guide star asterism -marked by the triangle, which is expected as tip-tilt and plate scales are not controlled in this region, and rotation effects are amplified, the stars being at larger radius.

- The computed position errors generally agree with the noise estimate, although there is some scatter. The bottom-right plot in the same figure shows the measured position error versus estimated noise, showing that the photon noise plays some role.

- Positions computed with this method lead to an estimate of the astrometric error of slightly over one milliarcsec.

Note that because of the way we compensate for plate scale mode changes between images, the position errors might be affected. For once, the larger position error due to noise on the fainter stars is going to propagate on all stars, including the brighter ones, which if noise is the dominant term, should have smaller position errors.

As said above, an alternative way to analyze these data, which is actually together more robust and more relevant to our purpose, is to look at the error on the star separations. Once all star positions have been determined, we can compute the separations of all stars with respect to all other stars $(12 \times 11 / 2$ pairs $)$, in each images (11 images), compute the standard deviation of all these separations and thus establish the probability density of the separation error versus the separation. These are presented in figure 7 . Each figure shows a contour plot of the said probability functions, together with a plot (solid line) of the median separation rms versus separation with associated error bars. The top two figures are for the full star sample, while the bottom two are for the brightest $50 \%$ of stars. The left two figures are for the unbinned data, i.e. each point is derived from a 15 seconds exposure image. In the right hand figures, we have binned the images by pair, i.e. 1 with 2 , 3 with 4, etc (with no overlap, i.e. not 2 with 3 as this would obviously bias the results). In these right hand figures, the dashed lines are just a re-plot of the solid lines of the left hand figures (individual 15s images), and the dotted lines are the dashed lines divided by $\sqrt{2}$. The excellent agreement between the unbinned case (dashed line) divided by $\sqrt{2}$ (dotted line) and the median for the binned $\mathrm{x} 2$ case (solid line) means the errors are coming from random sources, uncorrelated from image to image.

What are the possible error sources and what would be their signature on such a plot? Because of the random distribution of stars, photon noise should produce a flat distribution, that is, the separation error should be independent of the separation. A global plate scale error will produce an error which is linear with separation. Ripples in the surface of the high altitude DM(s) will produce bumps at the corresponding separations. If we concentrate on the lower right hand figure, what we see is a mix of these three components: It is mostly flat, 

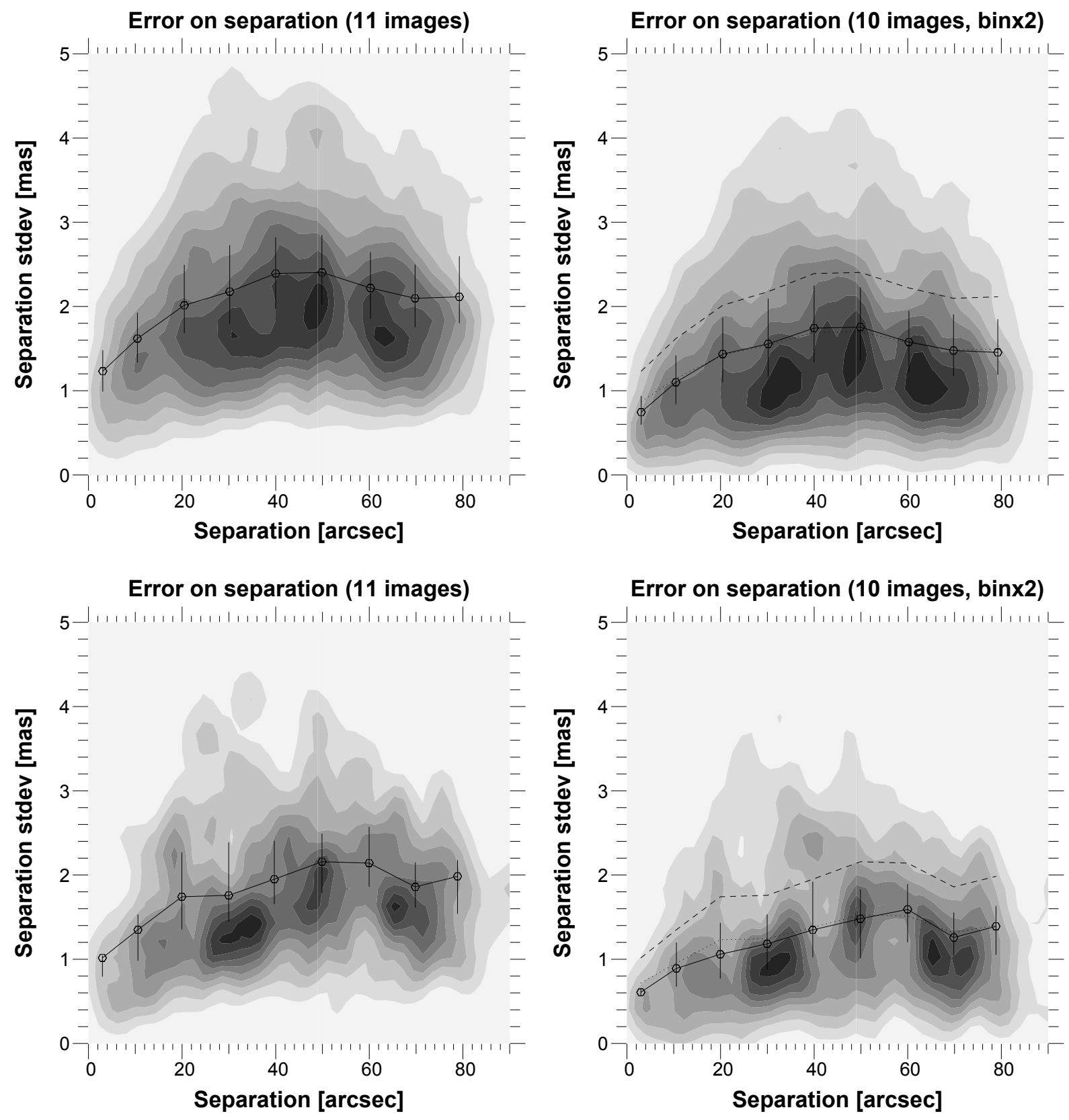

Figure 7. Astrometry with MCAO: Probability density of the separation error versus separation. Top: All stars. Bottom: $50 \%$ brightest stars in sample. Left: Using individual 15 second exposure images. Right: Using images combined by 2 (total exposure time 30 seconds). Solid lines and points are the median value per separation bin with 1 sigma error bar $( \pm 33 \%)$. See text for the meaning of the dashed and dotted lines.

with a slight increase of the error with separation, and may be a small bump around 60 arcsec or thereabout. We interpret this as being mostly dominated by noise, which will induce some plate scale error through the plate scale correction applied to the data as explained above. In effect, the two most important conclusions are:

- We see no evidence in our data set of distortion that would be induced (by CANOPUS) at timescales between $3 \mathrm{mn}$ (typical time between 2 images) and 45 minutes (elapsed time of the whole data set). If they are present, these distortion either evolve fast enough that they will average out in a typical astrometric 
data set, or are slow enough that they can potentially be calibrated out.

- Using averages of two images taken $3 \mathrm{mn}$ apart, the median separation error is of the order of 1.1 mas over the whole separation range. It is smaller for small separations, down to 600 micro-arcseconds for separation smaller than 5 arcseconds. Note that this is the error on the separation, so that providing enough references stars around the object of interest, this can be reduced by $\sqrt{2}$, leading to an astrometric error of 420 micro-arcseconds. From our reduced data set, there is no evidence that we reach any kind of systematics, so that further averaging will likely further reduce the astrometric error.

These results are very encouraging. Note however, that this study only applies to dynamic distortion issues. To deliver the full astrometric accuracy requires a precise and accurate calibration of the static distortion field of the instrument, which has not been done at the required level yet.

\subsection{Astrometric limitations and observing strategy}

It was recognized from the design phase that the two parabola optical design introduced significant field distortions (see upper right plot of figure 6). The plan was to calibrate the focal plane distortions with the required accuracy and perform the image distortion correction during data reduction. A twist to this problem was discovered during the commissioning phase: The distortion also affects the Tip-Tilt WFS focal plane. Thus when dithering, the TTGS constellation will be distorted (note that the TTWFS focal plane distortion at F/16 is different from the science focal plane distortions at F/33.2). The TT WFS assembly move as a whole/rigid body. Thus after a dither, the TTWFS are not matched with the TTGS positions anymore. These static position errors will eventually be compensated by the TT, plate scale and rotation offload to the Cassegrain rotator, inducing offsets, scalings and rotation in the output GSAOI field.

This problem was recognized early by the NFIRAOS (the TMT MCAO system) design team that solved it by using a four parabola optical relay system that has essentially zero distortions.

GeMS has to live with this shortcoming. The current mitigation plan involves finding an astrometric solution in the dithered images themselves, using as many reference (stars) as possible. There are only six parameters to determine ( 2 offsets, 3 plate scale modes and a rotation), so this should generally be do-able. This is what we used successfully in the astrometry analysis from the previous section. The same strategy could be used to reference images across nights or observing runs.

This technique will allow cross-referencing of the dithered images, but will not insure that the determined astrometric solution is the true one. This will require more calibrations, with a known, astrometry calibrated image or data of the same field. Note that the science image distortions induced by the TTWFS focal plane distortions depend not only on the constellation position, but obviously also on the constellation itself, so there is no way to easily calibrate on one object and apply for the next object. Solving this problem entirely would involve calibrating the full TTWFS focal plane distortion field. Even though, it would also mean being able to position blindly the TTWFS probes with an accuracy well below a milliarcsec, which was deemed from the beginning too difficult and expensive, as most of the shortcoming can be solved post-acquisition, as presented here. The problem of the huge dynamical range (typically 50000 to 200000) required from guide star position mechanisms is widely known in $\mathrm{AO}$ and has required dedicated hardware developments (NAOS, GMTIFS), but with the need to control plate scale/rotation in addition to simple position, MCAO raises the complexity of the problem.

\section{NEW PROBLEMS AND SOME SOLUTIONS}

This paper is primarily aimed to report on GeMS+GSAOI performance. However, in the latest part of commissioning, we have had to develop solutions to problems we encountered during the commissioning, and we would like to report them here. 


\subsection{Centroid gains determination with the Derviche mode}

Quadcells and the associated centroid gain problem are a pain. We have gone through many different trials to live-calibrate the ever changing centroid gains in the CANOPUS LGS WFS. Briefly, as this as described in more details in previous GeMS papers: the LGS WFS are using quadcells, i.e. 2x2 pixels in each subapertures. GeMS was designed in 1999 and at the time sub-electrons read-out noise devices did not exist. Now the technological landscape is different and there is no reason anymore to design SHWFS with quadcells. This section is thus somewhat obsolete, and you can safely skip it if not interested or concerned in centroid gain determination issues.

In any case, use of quadcell requires the knowledge of a calibration factor, the centroid gain, to transform the quadcell signal (unitless) into some meaningful quantity, say the spot position in arcsec. This centroid gain is proportional to the size and shape of the SHWFS spot, and thus in our case will change all the time with laser beam quality, seeing, etc... Many different methods were proposed for centroid gain on-line estimation in the past 20 years. Very early on, it was decided to use for GeMS the same dithering technique that is used successfully in Altair LGS: The LGS is moved along a circular path on the sky -"dithered", with a small 0.1" or less radiuswhich induces a very narrow spectral signature in the LGS position temporal spectrum. By performing a lock-in detection at the dithering frequency, one can easily measure how much is detected by the LGS WFS. Doing the ratio between the latter with the induced dithering amplitude directly gives the error on the centroid gain value. The lock-in method allows a good discrimination of the signal versus the background noise. The dithering, in the case of CANOPUS, was made with the Beam Transfer Optics Fast Steering Array (FSA), that is, the same mirrors used for the LGS stabilization on the LGS WFSs. ${ }^{10}$

However, consequent to constellation alignment problems and the finite range of the FSA, the steering mirrors often hit their range limits. When this happens, the modulation/dithering is interupted, with obvious catastrophic effects on the detection. This was also happening asynchronously between the LGS, inducing common and differential (between WFS) centroid gains estimation errors, with nefarious consequences on the system performance. Next we tried inducing this dithering with the TT mirror. This was working, and had the obvious advantage of using a common/single modulator, preventing the differential errors. However, the presence of vibrations in the system at about the dithering frequency required that we used a modulation which had some impact on the image quality (typically 20mas radius would have been needed).

Our new approach consists in using what we call the "derviche" mode: Instead of using the TT mirror, X and Y sinusoid spatial modes at the cut-off frequency of the DM are driven with 1/4 period delay between them. It turns out this will make the SH WFS spots turn clockwise in each "even" subaperture and anti-clockwise in "odd" subapertures. The use of this mode has multiple advantages: (a) For each WFS, the set of slopes can be spatially filtered before the lock in detection is performed by projecting on this spatial mode. This will filter out spot motion induced by, say, vibrations. (b) The Derviche modes are almost nonexistent in turbulence. Thus they are not controlled/reconstructed by our MMSE reconstructors. Which in turns means that their amplitude will not be affected by the close loop.

We have adopted this method as our baseline method. We lack a complete characterization of the fidelity of this method, however, it seems it is impervious to the obvious flaws of the other method and provide stable and consistent results.

\subsection{On-sky NCPA estimations}

In a MCAO system, the Non-Common-Path Aberrations have to be corrected over the entire extended compensated field of view simultaneously. New techniques have been developed for that purpose, primarily based on Phase Diversity (PD), which has the obvious advantages of measuring the aberrations exactly where it need to be corrected and to be very easy and cheap to implement. Results of NCPA compensation using Tomographic PD leading to average $\mathrm{H}$ band Strehl Ratio in excess of $85 \%$ over the $85 \times 85$ " of GSAOI have been reported elsewhere. ${ }^{16,17}$ In December 2011, we experimented with the use of these techniques on-sky. This required a slight modification of the PD minimization algorithms to include a turbulence residual term. Two diversity images were recorded sequentially with typically 30 seconds time separation. The phase diversity results made sense but the process did not converge. We attributed this to the instabilities in the centroid gains determination process, which at the time had not been upgraded with the derviche method (see section 4.1). This effort in 
on-going but we are confident it can lead to reliable results. How reliable and what are the environmental limits within which this method can be effectively applied (e.g. seeing) is still to be determined.

\subsection{On-sky misregistration calibrations}

We have successfully implemented and tested a method to measure the DM-to-LGSWFS misregistration onsky, similar to methods already proposed. ${ }^{18,19}$ It was important for us to make sure the complex LGS WFS mechanisms, or system flexure issues, were not inducing mis-registration from pointing to pointing or while following an object. Indeed, the LGS WFS mechanism are in constant motion to counteract the variation of range to the LGS, a consequence of the change in telescope elevation.

In the implemented method, a number of DM actuators ( 8 of them) are excited/dithered with sinusoidal amplitude of about 100nm peak-to-peak and a period of 4 to 8 times the loop sampling time. As for the Derviche mode above, we use a high spatial frequency mode that has a low variance in the turbulence, thus not a high gain in our reconstructor and in turn will not be efficiently corrected by the loop, leaving the signature intact for detection. We then acquire slope data (up to one minute of data, but 5 seconds seem to be enough), and Fourier filtering provides the slope variance at the dithering frequency. From there on, the amplitude of the slope variance in subapertures next to the dithered actuators can be used to determine the actuator position with respect to the 4 subaperture corners. Eventually, this leads to an estimation of the misregistration, magnification and rotation of the DM image on each of the five LGS WFS.

This operation is now done only occasionally, as the LGS WFS mechanisms have thus been proven to work reliably, with small enough hysteresis.

\section{CONCLUSION}

Results of ten years of efforts and one year of commissioning culminated in December 2011 with the first GeMS + GSAOI science images. Many data sets have been obtained with high PSF uniformity, fulfilling the promise of wide-field AO. Images with FWHM of 80 mas or better over the $85 \times 85$ arcsec field of view are now typically obtained under median seeing or better. GeMS + GSAOI has already broken several frontiers and records: Strehl ratio of $40 \%$ in $\mathrm{H}$ band have been demonstrated, we believe the highest to date with a LGS-based AO system on a large telescope, usually limited by focus anisoplanatism at this wavelength.

We also presented the first analysis of the astrometric properties of MCAO compensated images, showing very encouraging results: we do not detect any detrimental effects on the astrometry from the MCAO compensation. Astrometric errors of well below a milliarcsec should be reachable (we demonstrate 420 microarcsecond).

The compensation performance is currently limited by servolag -a consequence of the low photon return,-generalized fitting - a consequence of the temporary absence of the CANOPUS mid-altitude DM,-- and static aberrations. Work is continuing on the system to improve on all these aspects.

GeMS + GSAOI is a unique instrument, and will no doubt deliver first class science. It will be offered to the daring Gemini astronomers sometime in 2013.

\section{ACKNOWLEDGMENTS}

Part of this work has been founded by the Gemini Observatory. The Gemini Observatory is operated by the Association of Universities for Research in Astronomy, Inc., under a cooperative agreement with the NSF on behalf of the Gemini partnership: the National Science Foundation (United States), the Science and Technology Facilities Council (United Kingdom), the National Research Council (Canada), CONICYT (Chile), the Australian Research Council (Australia), Ministério da Ciência e Tecnologia (Brazil) and Ministerio de Ciencia, Tecnología e Innovación Productiva (Argentina). 


\section{REFERENCES}

1. Bec, M. et al., F. R., "The gemini mcao bench: system overview and lab integration," Proc. SPIE 7015, 701568 (2008).

2. Boccas, M. et al., F. R., "Gems: Gemini mcao system, current status and commissioning plans," Proc. SPIE 7015, 70150X (2008).

3. D'Orgeville, C. et al., F. D., "The gemini south mcao laser guide star facility: getting ready for first light," Proc. SPIE 7015, 70152P (2008).

4. Neichel, B., Rigaut, F., et al., M., "The gemini mcao system gems: nearing the end of a lab-story," Proc. SPIE 7736, 7736 (2010).

5. Rigaut, F., Neichel, B., Bec, M., Boccas, M., Garcia-Rissmann, A., and Gratadour, D., "A sample of gems calibrations and control schemes," in AO4ELT-1, Clenet, Y., Conan, J.-M., Fusco, T., and Rousset, G., eds., EDP Sciences 1, 08001 (2010).

6. Neichel, B., Rigaut, F., Matthieu, B., and Aurea, G.-R., "Reconstruction strategies for gems," in AO4ELT-1, Clenet, Y., Conan, J.-M., Fusco, T., and Rousset, G., eds., EDP Sciences 1, 02010 (2010).

7. Ellerbroek, B.L., "First-order performance evaluation of adaptive-optics systems for atmospheric-turbulence compensation in extended-field-of-view astronomical telescopes," JOSA-A 11, 783 (1994).

8. Rigaut, F., Ellerbroek, B., Flicker, R., "Principles, limitations, and performance of multiconjugate adaptive optics," Proc. SPIE 4007, 1022 (2000).

9. Neichel, B., Rigaut, F., Arriagada, G., et al, "Status of the Gemini MCAO System GeMS", Proc. SPIE 8447, 8447-32 (2012).

10. dOrgeville, C., Diggs, S., Fesquet, V., Neichel B., et al, "Gemini South multi-conjugate adaptive optics (GeMS) laser guide star facility on-sky performance results," Proc. SPIE 8447 (2012).

11. Neichel, B., Parisot, A., Petit, C., Fusco, T. and Rigaut, F., "Identification and calibration of the interaction matrix parameters for AO and MCAO systems," Proc. SPIE 8447 (2012).

12. Neichel, B., Callingham, J., d'Orgeville, C. et al, "Characterization of the Sodium Layer at Cerro Pachon, and impact for GeMS performance," Proc. SPIE 8447, 8447-176 (2012).

13. Carrasco, E.R., Edwards, M., McGregor, P.J., Winge, C., et al, "Results from the commissioning of the Gemini South Adaptive Optics Imager (GSAOI) at Gemini South Observatory," Proc. SPIE 8447 (2012).

14. Marchetti, E., Brast, R., Delabre, B., Donaldson, R., Fedrigo, E., Frank, C. et al, "On-sky Testing of the Multi-Conjugate Adaptive Optics Demonstrator," The Messenger, 129, 8-13 (2007).

15. Ragazzoni, R., Diolaiti, E., Farinato, J., Fedrigo, E., et al, "Multiple field of view layer-oriented adaptive optics," A\&A 396, 731-744 (2002)

16. Rigaut, F., Neichel, B., et al., "Gemini south mcao on-sky results," in 2nd AO4ELT conference - Adaptative Optics for Extremely Large Telescopes, Véran, J., Clénet, Y., and Fusco, T., eds., EDP Sciences 2 (2012).

17. Gratadour, D., Rigaut, F., Neichel, B., "Tomographic phase diversity for phase retrieval on wide-field AO systems," in AO4ELT-2, Véran, J., Clénet, Y., and Fusco, T., eds., EDP Sciences 2 (2012).

18. Esposito, S., Tubbs, R., Puglisi, A., Oberti, S., Tozzi, A., Xompero, M., Zanotti, D., "High SNR measurement of interaction matrix on-sky and in lab," Proc. SPIE 6272, 62721C (2006).

19. Béchet, C., Thiébaut, E., Tallon, M., Kolb, J., Madec, P-Y., "Identification of system misregistrations during AO-corrected observations," [AO4ELT-2], Véran, J., Clénet, Y., and Fusco, T., eds., EDP Sciences $2(2012)$. 